\title{
Ciencia y tecnología para mejorar la calidad de vida en poblaciones rurales pobres del Perú
}

\author{
Alfredo Oliveros Donohue \\ Programa Euro-Solar, Perú
}

Ingeniería Industrial n. 28, 2010, ISSN 1025-9929, pp. 211-226

Recibido: 17 de junio del 2010 / Aprobado: 13 de julio del 2010

RESUMEN: El presente artículo pone de relieve la relación existente entre calidad de vida y energía, que se acentúa en el ámbito rural, y cómo la primera puede mejorar con la influencia de la ciencia y la tecnología que se han desarrollado en nuestro país, si estas son utilizadas tomando en cuenta el concepto de 'energización'. La articulación adecuada entre la oferta y la demanda de energía que las nuevas tecnologías exigen se explica en el concepto de energización, que, entre otros, enfatiza el uso productivo de las energías renovables, para añadir valor a los productos naturales a través de mecanismos de producción y comercialización gestionados por las propias comunidades. Finalmente, el artículo muestra también las transferencias de tecnologías apropiadas y limpias a las micro y pequeñas empresas exitosas.

Palabras clave: valor agregado / calidad de vida / población rural / Andes Perú /energía renovable

\section{The use of science and technology to improve the quality of life of the rural poor in peruvian population}

ABSTRACT: This article paper highlights the relationship between energy access and quality of life and how investment in science and technology can improve the quality of life in rural areas of Peru, under a new energy framework, the possibility of improving the welfare of the rural poor in Peru is described. The energy framework describes how appropriate technologies when linked to energy demand and put to productive or commercial use can enable communities to become more self-sufficient and less reliant on external resources to improve their lives. Finally, the paper also shows how the transfer of appropriate clean technologies and micro/small enterprises can be successful.

Keywords: value added / quality of life / rural population/ Andes Peru / renewable energy 


\section{INTRODUCCIÓN}

Una característica de las poblaciones rurales pobres en nuestro país es el limitado acceso a servicios públicos de calidad: luz, agua, transporte, educación, comunicación, salud, entre otros.

La actividad económica en la que se sustenta su calidad de vida es la producción de bienes y servicios básicos vinculados a los sectores agrícola, pecuario y minero. Participan también en la artesanía, que recoge el legado de las tradiciones y costumbres de nuestras poblaciones.

Lo anterior determina que la producción de estos sectores gire en torno a productos naturales de bajo valor, que son acopiados y colocados en los mercados por los intermediarios, quienes en la mayoría de los casos son los que obtienen mayores beneficios del intercambio, que se realiza en lugares establecidos mediante las ferias locales.

Investigaciones realizadas por diversos científicos concluyen que la calidad de vida se puede expresar en función de parámetros de desarrollo, como: consumo de proteínas, vitaminas, analfabetismo, niños muertos al nacer, expectativa de vida, con lo que se estructura el índice de calidad de vida para cada país (o población).

Posteriormente, se ha encontrado que existe una relación muy clara entre el índice de calidad de vida y el consumo de energía per cápita de una sociedad determinada, conforme se aprecia en la figura 1.

Figura 1

Relación entre la calidad de vida y el consumo de energía

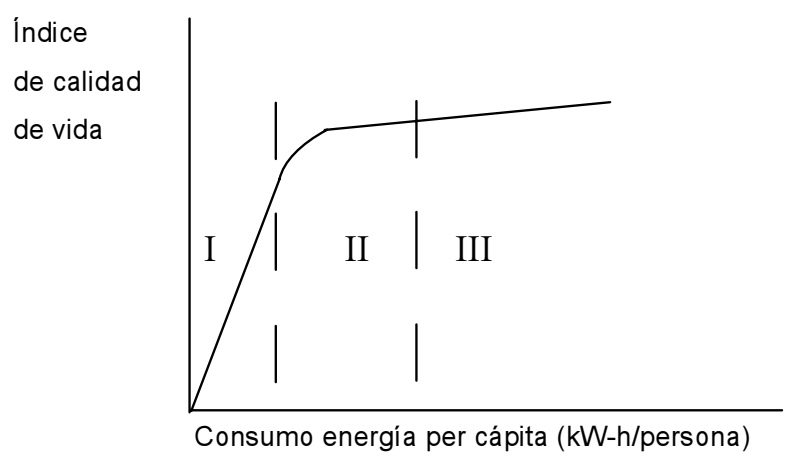

Fuente: Programa de las Naciones Unidas para el Desarrollo 1992, 1993, 1994. Elaboración propia. 
Aquí se puede ver que en las sociedades de menor desarrollo, la relación es muy clara y directa (I). En las ex socialistas hay una inflexión (II) y en las industrializadas la incidencia del consumo de energía en el índice de calidad de vida es mínimo (III).

Este artículo se ha escrito a partir de las observaciones y experiencias realizadas por el autor en zonas rurales pobres de nuestro país y pretende entregar propuestas de aplicación de ciencia y tecnología (CyT) para mejorar la calidad de vida de estas. Asimismo, busca presentar casos exitosos sobre el uso de la energía renovable y las tecnologías limpias para lograr este propósito.

\section{MÉTODO}

Por décadas, las prácticas de electrificación han sido tomadas del mundo industrializado en países por lo general con alta densidad poblacional y con una geografía más homogénea, y se han aplicado en nuestro país sin la menor adaptación a nuestras propias características. Esta aproximación a la electrificación se basa en el aumento de la oferta a través de la extensión de redes o en la instalación de grupos estacionarios, cuyo principal propósito ha sido la iluminación de las comunidades.

Este procedimiento, que no obstante puede reportar inicialmente altos índices de utilización, a menudo queda grande. Por un lado, porque no favorece el desarrollo de la región, y por otro, porque no permite al usuario que tiene ingresos irregulares y escasos afrontar una tarifa creciente.

Se presenta aquí un método alternativo para introducir energía, que ha sido aplicado por el autor en zonas rurales del Perú y Bolivia, denominado "energización". Esta propuesta se basa en la idea de que la necesidad de energía va más allá de la necesidad de electricidad. Considera también otras formas de suministro de energía, tales como la térmica y la mecánica. Además, en la determinación de la mejor manera de suministrar energía, tiene en cuenta factores sociales y económicos como:

- Tecnología apropiada de bajo costo.

- Uso productivo de la energía generada para suministrar valor agregado a los productos naturales de la zona. 
- Mejora de la capacidad gerencial de las comunidades.

- Uso de fuentes renovables de energía.

- Fortalecimiento de la organización y valores ancestrales de la población, como la solidaridad, el trabajo comunitario y el respeto del medio ambiente.

De esta manera, siempre será posible brindar energía a la comunidad a un precio menor que el suministrado por la red, con períodos cortos de recuperación del capital con respecto a los grandes proyectos. Esta recuperación rápida del capital es posible cuando la energía es usada para generar ingresos por los usos finales adicionales, que no son posibles cuando la electricidad es vendida a las comunidades solamente para la iluminación pública y doméstica.

Figura 2

Esquema del concepto de 'energización'

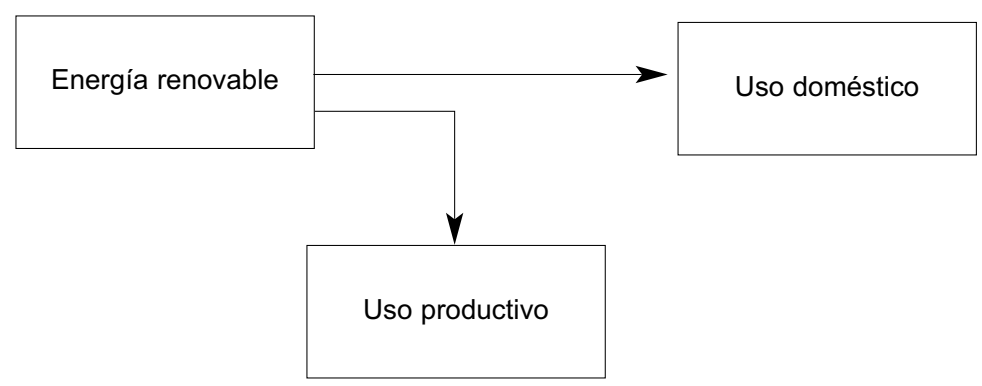

Fuente: Oliveros 1998.

\section{LOS PROYECTOS}

Para su mejor ubicación se ha considerado la oferta de los distintos proveedores de energía, como el sol, el viento, la micro hidro y la biomasa. Estos pueden presentarse tanto solos como agrupados o asociados con la energía comercial, en cuyo caso se hace un dimensionamiento económico de los equipos que las van a utilizar. 


\subsection{La oferta de energía}

La ONG Energía, Desarrollo y Vida (Edevi) realizó varios proyectos en comunidades rurales del departamento de Puno, utilizando el concepto de energización. Inició la construcción de centrales hidroeléctricas con equipamiento de procedencia nacional en todas las comunidades cuyos nombres aparecen a continuación (solo en Pahara la Dirección Ejecutiva de Proyectos del Ministerio de Energía y Minas (DEP/MEM) terminó las obras que inició Edevi):

- Ayapata $100 \mathrm{~kW}$, con turbina Pelton, noviembre de 1998.

- Coaza $100 \mathrm{~kW}$, con turbina Michel Banki, marzo de 1994.

- Pahara 150 kW, con turbina Francis, noviembre de 1995.

Pero solo en la primera de las nombradas se realizó la aplicación productiva en el marco de un proyecto de energización que se tituló "Mini complejo productivo de Ayapata", que fue apoyado por el Fondo de Contravalor Perú-Suiza. En esta comunidad se construyó, en un área de $5.000 \mathrm{~m}^{2}$, el referido minicomplejo donde se instaló una panadería, un cargador de baterías, un molino de granos y una sección para producir harina de papa, con la perspectiva de producir harina de este tubérculo y comercializarla como sucedáneo para la elaboraboración de pan. Para la gestión de la infraestructura energética y productiva se formó una cooperativa (en la actualidad con más de 15 años de existencia), que en consenso estableció 3 rangos de tarifa a la que accedían los vecinos, de acuerdo con sus ingresos y la actividad económica, logrando que la morosidad sea mínima.

Otras instituciones, como el Intermediate Technology Development Group (ITDG) (Inglaterra), la Universidad Nacional de Ingeniería, la Empresa de Administración de Infraestructura Eléctrica (Adinelsa) y la Dirección General de Electrificación Rural (DGER/MEM) han trabajado en rangos menores, con diversos energéticos: sol, vientos, pequeñas caídas de agua, logrando instalaciones confiables, que son fabricadas por empresas nacionales. Debemos mencionar otra tecnología interesante: la producción de biodiésel. La Universidad Nacional Agraria La Molina, con el auspicio del Concytec, logró producirlo en una planta piloto. Finalmente, también existe la generación de electricidad a partir del biogás, desarrollada, a escala piloto, por un equipo de profesores de la Universidad Nacional de Ingeniería. 


\subsection{La demanda de energía}

\subsubsection{La demanda doméstica}

Es la más común. Se utiliza para dar comodidad pero no reporta los ingresos que una sociedad necesita para capitalizarse. Hay que destacar los avances que se han logrado para satisfacer la demanda con los menores consumos; es el caso, por ejemplo, de los focos ahorradores y los electrodomésticos de muy bajo consumo.

\subsubsection{La demanda productiva}

La demanda de energía es muy variada, y en los casos escogidos la población ya hace esta aplicación pero con una tecnología muy rudimentaria que no produce ingresos significativos para los grupos que la practican.

\subsubsection{Proyecto productivo auspiciado por la OEA}

En la búsqueda de aplicaciones que pudieran ayudar a lograr este propósito, la OEA, en coordinación con el Concytec, ejecutó el proyecto "Programa horizontal de tecnologías limpias y energías renovables", en el que participaron nueve países: Argentina, Ecuador, El Salvador, México, Panamá, Paraguay, Perú, Trinidad y Tobago y Surinam. Tuvo como propósito que las pequeñas y medianas empresas de las poblaciones más pobres de los países participantes en el proyecto, adopten procesos de producción más limpios y utilicen energías renovables.

Los sectores priorizados para el proyecto fueron:

- agroindustria-lácteos,

- curtiembre, y

- residuos sólidos y efluentes líquidos.

Como parte de las actividades del plan de ejecución del proyecto se realizaron talleres de transferencia de tecnologías limpias para pymes de cada uno de los sectores mencionados en los que intervinieron empresarios, consultores e investigadores de los países participantes.

El Taller de Agroindustria-Lácteos se realizó en Piura y contó con la participación de un experto uruguayo, uno francés y cuatro piura- 
nos, quienes trabajaron estrechamente con los extranjeros preparando el manual que se utilizó durante la transferencia de tecnología. Para la realización del componente práctico del taller se recibió el apoyo de la Universidad Agraria La Molina, la Universidad Nacional de Piura, el Instituto Superior Tecnológico de Sullana y la empresa La Cabrita. La réplica de esta transferencia se realizó en Cajamarca, con un experto peruano y uno francés, con el apoyo del Senati.

El Taller de Curtiembre se desarrolló en Arequipa y contó con la participación de un experto mexicano, uno francés y dos consultores locales, que trabajaron con los extranjeros preparando el manual y el material que se utilizó durante la transferencia. Para la realización del componente práctico del taller se contó con el apoyo de una planta de curtido de la ciudad y de la planta piloto de la Universidad de Arequipa. La réplica de esta transferencia se efectuó con un experto peruano en una empresa de Ayacucho, con el apoyo de la Universidad San Cristóbal de Huamanga.

El Taller de Residuos Sólidos y Efluentes Líquidos se realizó en la ciudad de Panamá y contó con la participación de un experto peruano y tres locales, que trabajaron en la preparación del manual y el material que se utilizó durante la transferencia.

Para la realización del componente práctico del taller se contó con el apoyo de una granja para crianza de cerdos. La réplica de esta transferencia se realizó con un experto peruano y uno francés en una empresa de Piura, con el apoyo de la Universidad Nacional de Piura.

\subsubsection{Proyecto auspiciado por el PNUMA}

El Concytec trabajó también en el desarrollo de tecnologías limpias con el apoyo del PNUMA, logrando otro grupo de estas:

- Planta de colorantes y aceites esenciales.- Cuando se empieza el programa de tecnologías limpias se encuentra que existía una planta desarrollada por profesionales del Concytec, que se encontraba sin uso en su local institucional; por ello, se convoca a un concurso en el que resulta ganadora la Universidad Agraria de la Selva, ubicada en Tingo María.

- Extracción de oro sin usar mercurio.- Iniciativa presentada por un profesor investigador de la UNI, proyecto que el Concytec decide respaldar en el marco de su programa de apoyo al investigador. 
- Producción de biodiésel a partir de aceites usados.- Iniciativa presentada por un profesor investigador de la Universidad Nacional Agraria La Molina.

- Uso del biogás en motores gasolineros y petroleros.- Proyecto exitoso que se desarrolló en la UNI y fue presentado al concurso de proyectos de investigación del Congreso Nacional de Ingeniería Mecánica Eléctrica y Ramas Afines (Conimera), en el que otuvo el primer puesto.

- Generación de electricidad híbrida.- Iniciativa de profesores de la Universidad Pedro Ruiz Gallo,

- Producción de tejidos con hilos de algodón de color natural.- Iniciativa desarrollada por profesores del Senati.

\section{RESULTADOS}

\subsection{Tecnología energética}

En nuestro país existe una importante experiencia en la construcción de todos los componentes electromecánicos para generar electricidad con caídas de agua. En las fotos que siguen se pueden apreciar algu-

\section{Foto 1}

Minicentral hidroeléctrica de Coaza, construida por Edevi en un pequeño taller de metalmecánica de Lima

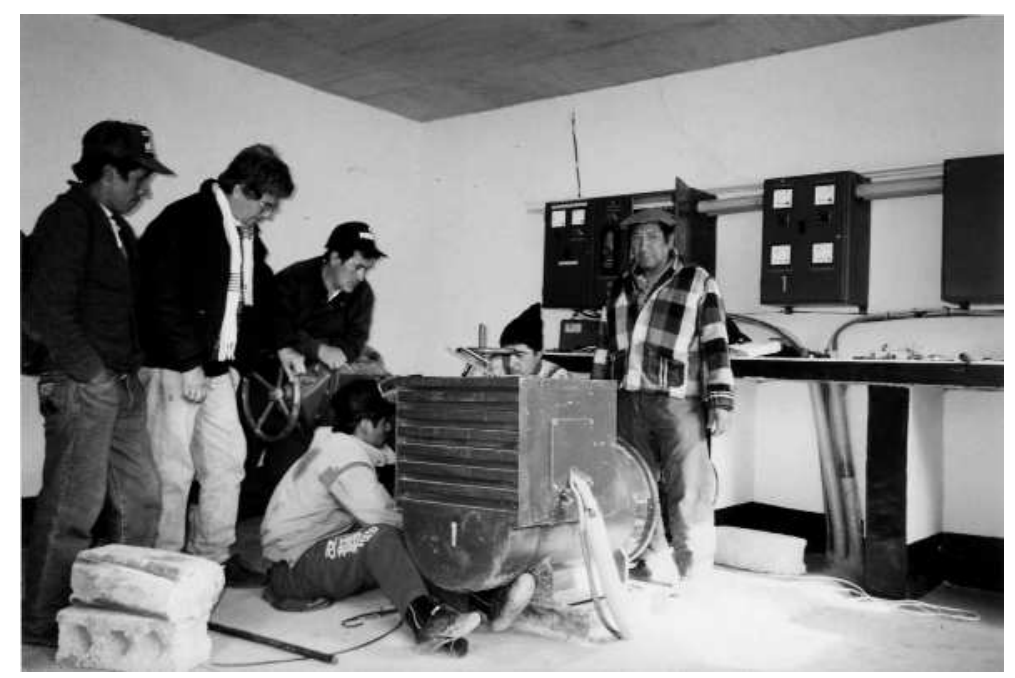

Foto del autor. 
nas instalaciones realizadas con diversos energéticos. El ITDG (Intermediate Technology Development Group) realizó una importante transferencia de tecnología para fabricar microturbinas hidráulicas.

En lo que respecta a la generación de electricidad fotovoltaica, en la foto 3 se presenta una instalación fabricada por el Centro de Energía Renovable de la UNI, con el auspicio de la DGER/MEM, en la comunidad campesina Vilcallamas Arriba, en Puno.

\section{Foto 2}

Minicentral hidroeléctrica construida por el ITDG en un pequeño taller de Cajamarca

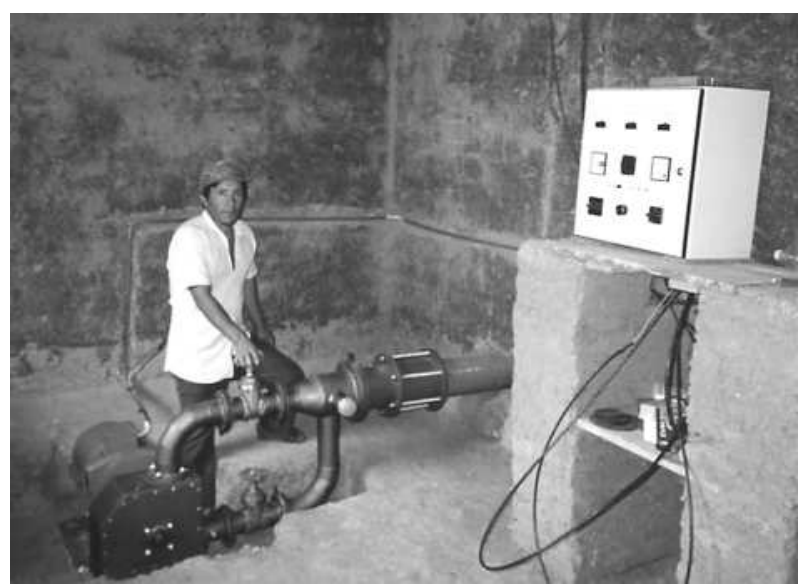

Foto del autor.

Foto 3

Generador fotovoltaico de 2 kW instalado en Vilcallamas Arriba, Puno

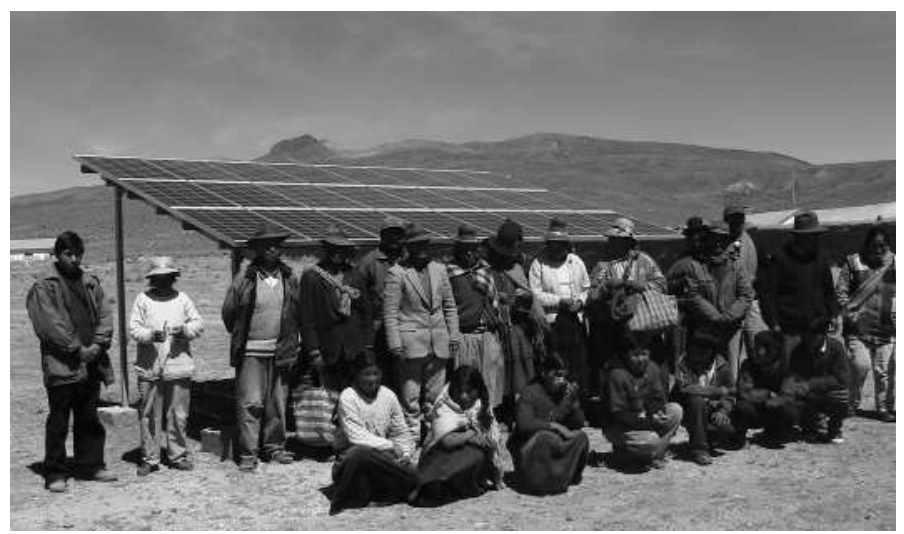

Foto: DGER/MEM. 
Con la electricidad generada se ha logrado darle valor agregado a la fibra de alpaca, al elaborar diversos productos como chalinas, chompas, ponchos, frazadas, que ahora se comercializan en las ferias locales. Por ejemplo, el precio de las chalinas pasó de 1,0 US $\$ / \mathrm{kg}$ de fibra, que la pagaba el acopiador, a 7 US $\$ / \mathrm{kg}$.

Otro tema es la producción de biodiésel, tecnología que puede ser de gran uso en nuestro país. En la foto 4 se aprecia una planta que puede producir 1.000,00 lts/día. Esta planta funciona con aceite usado del comedor de estudiantes de la UNALM, pero se han hecho estudios para trabajarla con otros energéticos, como el aceite de palma en el caso de nuestra Amazonía.

\section{Foto 4}

Planta piloto desarrollada en la UNALM para producir biocombustible con tecnología limpia a partir de desechos de su comedor universitario

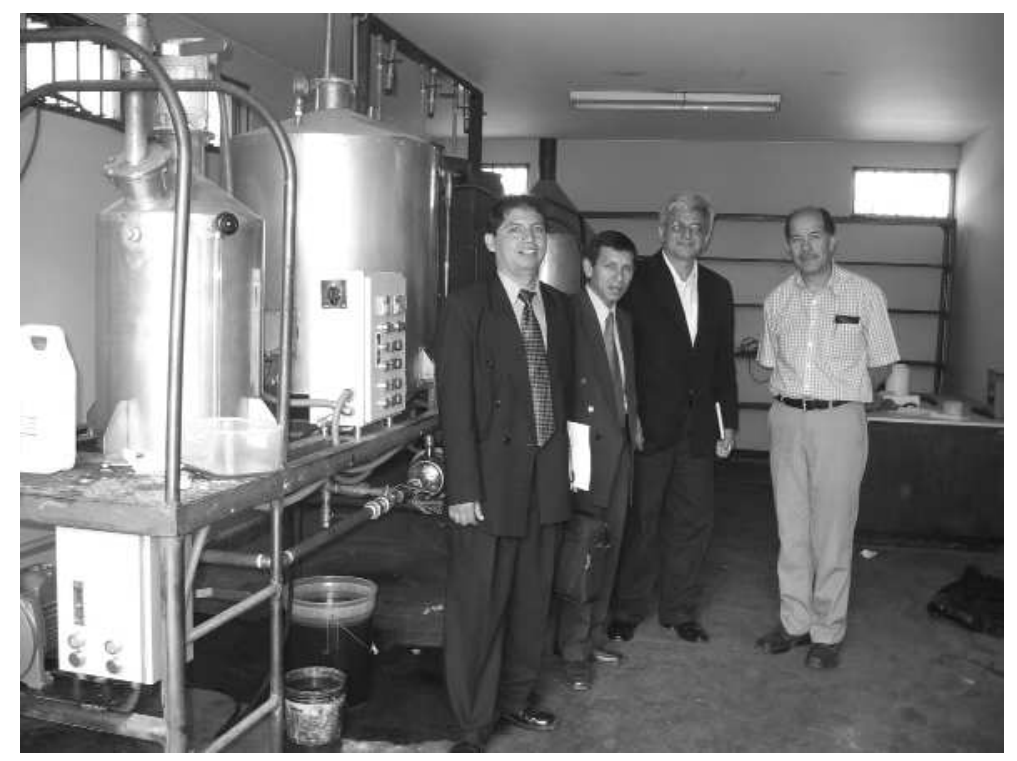

Foto del autor.

La generación de electricidad a partir del biogás es una interesante alternativa que se logró en la Facultad de Ingeniería Mecánica de la UNI, trabajando de manera coordinada con las facultades de Química y de Ambientales. 
Se comenzó modificando los motores gasolineros para que trabajen con el biogás obtenido, primero, de una planta piloto que el ex Itintec dejó en un local de Villa El Salvador (ahora propiedad del Senati). Luego de lograr los primeros resultados el experimento se trasladó a la planta de tratamiento de aguas servidas Unitrar, de la Facultad de Ambientales de la UNI. En esta existe un reactor anaerobio de flujo ascendente (RAFA), que trata las aguas servidas de tres asentamientos humanos antes de ingresar a la laguna de oxidación.

Durante el proceso arriba señalado se generaba biogás $\left(15 \mathrm{~m}^{3}\right.$ al día en el verano y $5 \mathrm{~m}^{3}$ al día en invierno), con el que se trabajó la modificación del motor, así, se generaron $2.0 \mathrm{~kW}$ de electricidad a 220 $\mathrm{V}$ y $60 \mathrm{~Hz}$, por aproximadamente 5 horas. El generador mantuvo su sistema de arranque y se montó un carburador auxiliar, que entraba en funcionamiento una vez que arrancaba el motor, logrando una generación eléctrica con voltaje y frecuencia constante. Ganó un premio pero hasta el momento la tecnología no ha sido transferida.

En la foto 5 se puede apreciar el generador prototipo donde los focos de $100 \mathrm{w}$ se prendían por filas de 5 .

\section{Foto 5}

Generación de electricidad a partir del biogás con motores gasolineros modificados

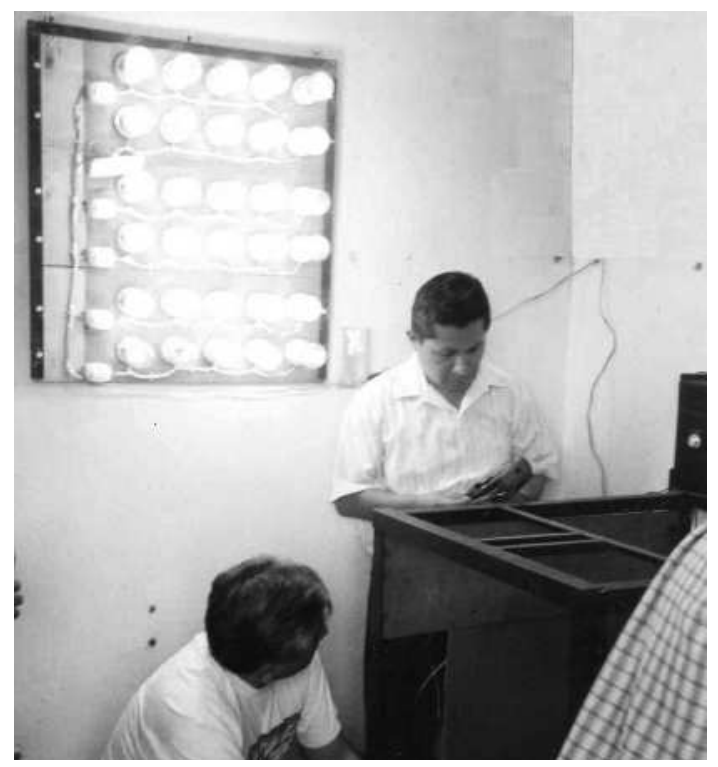

Foto del autor. 
Luego de esta experiencia se pasó el conocimiento a un motor Diésel de $6 \mathrm{HP}$, con el que también se logró generar electricidad de una manera estable.

\subsection{Producción de quesos}

Una posibilidad muy interesante en nuestro país es la relacionada con la elaboración de productos basados en la leche de cabra, si consideramos que existen más de 2,0 millones de cabezas y más de 210.000 criadores, que son en su mayoría campesinos de muy bajos ingresos.

Estos criadores producen quesos muy simples, con muy bajo valor en los mercados $(\mathrm{S} / .5,0 / \mathrm{kg})$. Con algo de ayuda y difusión, podrían producir otras variedades y obtener mejores precios, generando más ingresos para sus familias y comunidades.

\section{Foto 6}

\section{Réplica de la transferencia de tecnología realizada en Cajamarca}

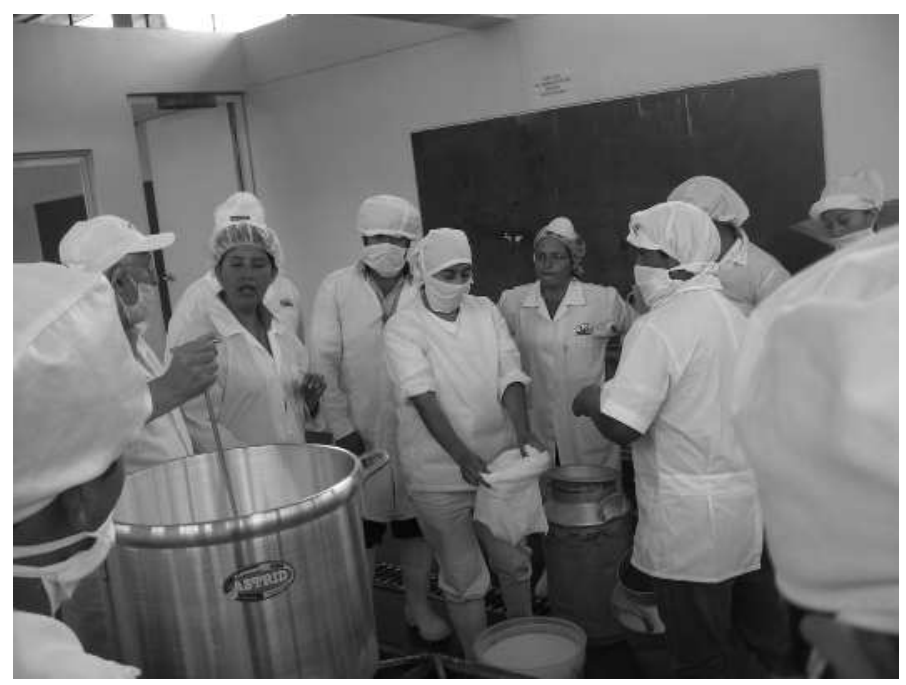

Foto del autor. 
Esta actividad comenzó con las pymes del valle del Chillón, donde asistieron unas 20 personas que aprendieron a preparar quesos mejorados. Luego, el taller se llevó a cabo en Piura, departamento considerado como el primer productor de ganado caprino, y finalmente se trabajó en Cajamarca, con la asociación de productores de lácteos.

En todos los casos se contó con la presencia de empresarios de las pymes, la universidad local y expertos nacionales y extranjeros.

\subsection{Extracción de oro sin utilizar mercurio}

Esta tecnología responde a una preocupación del sector minería por los pequeños mineros de oro, sobre todo en Madre de Dios, pero podría servir también para los de Carabayllo, quienes extraen oro pero afectando el medio ambiente, motivo por el cual el Concytec auspició esta tecnología.

Foto 7

Planta para la extracción de oro sin usar mercurio

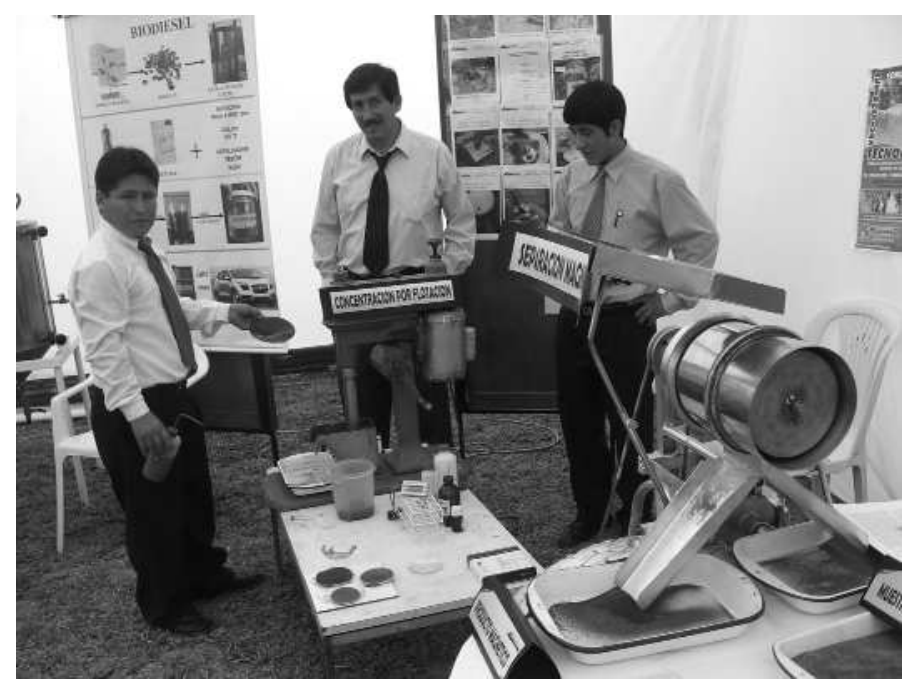

Foto del autor. 


\subsection{Hilos de color natural}

Este singular algodón data de la cultura preínca y es promovido por el Ministerio de Industria, Turismo, Integración y Negociaciones Comerciales Internacionales (Mitinci), habiéndose recuperado importantes hectáreas de cultivo en el norte de nuestro país. No obstante, el hilo se elabora de manera rudimentaria, mediante el uso de la rueca.

Para tener un procedimiento mejor, el Concytec auspició al Senati para que desarrolle un tren de producción de prendas, que incluye el hilo, el urdido y el telar, lográndose aumentar la productividad de 30 $\mathrm{cm} /$ día a $700 \mathrm{~cm} /$ día.

En la foto 8 se puede observar uno de los trenes construidos por el Senati, que son ofrecidos a los interesados en sus unidades de capacitación.

Aquí se destaca el cambio de la tecnología tradicional para producir los hilos, al pasar de la rueca a la hiladora eléctrica. Mediante esta se obtienen hilos de mejor calidad al hacerlo con mayor torsión y espesor uniforme, con lo que se consigue más resistencia. Con hilos de menor calibre y mayor resistencia se obtienen prendas de calidad superior y, por lo tanto, de mejores precios.

\section{Foto 8}

Tren de producción de hilados

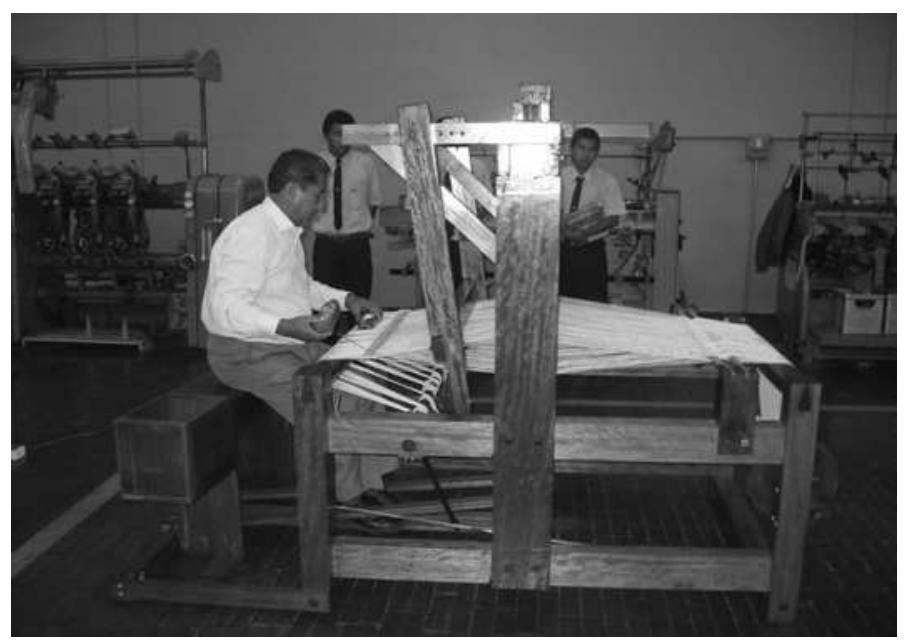

Foto del autor. 


\section{LAS PROYECCIONES}

De acuerdo con la experiencia de Ayapata, una combinación adecuada de oferta renovable de energía con tecnologías de producción limpias, pueden contribuir a producir riqueza que bien utilizada originará una mejor calidad de vida para las poblaciones rurales pobres, generando excedentes que les permitan ofrecer la iluminación con tarifa escalonada, a un costo tal que todos los pobladores puedan hacer uso de ella. Esto es posible cuando en las propias comunidades hay una organización adecuada para realizar aplicaciones comunitarias y establecer características de gestión en consenso.

Estas experiencias se pueden replicar en muchos lugares de nuestro país, donde existan recursos energéticos renovables y productos naturales a los que se pueda dar mayor valor agregado.

\section{CONCLUSIONES}

- En las sociedades menos desarrolladas el consumo de energía guarda una relación casi lineal con el índice de calidad de vida.

- Es posible poner el conocimiento al servicio de las comunidades rurales pobres con la participación de universidades, ONG, investigadores empresarios y el propio Estado.

- En el concepto de energización, la energía es el articulador entre la oferta y la demanda.

- Se logra un aumento del valor de los productos naturales cuando se emplea tecnología apropiada y limpia, en el lugar donde existe el recurso natural.

- El conocimiento está en manos de los especialistas que lo generaron y no existen mecanismos claros de transferencia ni de apoyo.

- Las transferencias se realizaron en diversas regiones de nuestro país con especialistas extranjeros y nacionales.

- Los proyectos se financiaron con recursos del Estado y de la cooperación internacional.

Se recomienda:

- Actualizar y consolidar el conocimiento generado.

- Estructurar los programas de desarrollo, la transferencia de tecnologías y la asistencia técnica para las zonas pobres de nuestro país. 
- Transmitir este conocimiento a los lugares donde existen recursos naturales similares a los utilizados con las tecnologías descritas, con potencial comercial.

- Expandir el conocimiento para crear nuevas tecnologías apropiadas y limpias que potencien la megadiversidad existente en nuestro país.

- Estudiar la posibilidad de considerar el proceso de energización mostrado, como alternativo a la electrificación rural.

- Promover la participación del sector privado en la financiación de los proyectos.

\section{BIBLIOGRAFÍA}

Adinelsa. "Energía solar". <http://www.adinelsa.com.pe>. [Consulta: 29 de mayo del 2010.]

Dirección General de Electrificación Rural. <http://www.dger. minem.gob.pe>. [Consulta: 29 de mayo del 2010.]

Muguerza, Jorge (2005). Elaboración del diseño de un programa piloto de financiamiento de transferencia de tecnologías limpias a la pequeña y mediana empresa. Lima: UNEP/Concytec.

Oliveros, Alfredo (febrero de 1998). "Energising rural areas of Peru". Energia News. Vol. 2, Issue1. Leusden: Energia, the International Network on Gender and Sustainable Energy.

Perrone, César (2006). Manual de tecnologías limpias para pymes del sector agroindustrial (lácteos). Lima: Concytec.

Rodríguez, Arturo (marzo del 2006). Manual de tecnologías limpias para pymes del sector curtiembre. Lima: Concytec.

Sandoval, Leandro (octubre del 2006). Manual de tecnologías limpias para pymes del sector residuos sólidos. Lima: Concytec.

Soluciones prácticas-ITDG. "Energías renovables". <http://www.solucionespracticas.org.pe>. [Consulta: 22 de mayo del 2010.]

Suárez M., Jorge. "Electrificación rural con energías renovables". Asociación Electrotécnica Peruana. <http://www.aep-peru.org>. [Consulta: 22 de mayo del 2010.] 